\title{
Uso de baixo volume oleoso e assistência de ar no controle de plantas daninhas
}

\section{Use of low volume in oil and air assistance in weed control}

\author{
Emerson da Silva Teixeira ${ }^{1 *}$; Jethro Barros Osipe ${ }^{1}$; Ulisses Delvaz Gandolfo"; \\ José Francirlei Oliveira ${ }^{2}$; Marco Antonio Gandolfo ${ }^{3}$; Robinson Osipe ${ }^{3}$
}

\section{Resumo}

O controle das plantas daninhas é uma ação de grande importância para a preservação do potencial produtivo das culturas. O objetivo deste trabalho foi avaliar a eficiência do controle de plantas invasoras nas aplicações terrestres de herbicidas com e sem assistência de ar, utilizando volumes convencionais de pulverização comparados com baixo volume oleoso (BVO). O delineamento experimental empregado foi inteiramente ao acaso, com parcelas de $48 \mathrm{~m}^{2}$, com 5 tratamentos e cinco repetições. A aplicação ocorreu em pós-emergência de Digitaria insularis (L.) Fedde e Commelina benghalensis L. Os tratamentos consistiram de pulverização convencional e com sistema BVO, ambos com e sem assistência de ar, mais uma testemunha. As aplicações com sistema BVO sem assistência de ar mostraram níveis de controle semelhantes às aplicações convencionais. $\mathrm{O}$ uso da assistência de ar elevou a eficiência das aplicações convencionais e reduziu a eficiência das aplicações em BVO.

Palavras chave: Pulverização, planta daninha, eficiência de controle

\begin{abstract}
Weeds control is an important action to keep the crop production potential. The objective of this study was to evaluate the efficiency of weed control in ground spraying with and without air assistance, using conventional flow rate in herbicide application compared to low volume in oil (LVO). The experimental design was randomized with $48 \mathrm{~m}^{2}$ per plot, and five replications of five different treatments. The applications occurred in post-emergency of Digitaria insularis (L.) Fedde and Commelina benghalensis L. The treatments were of conventional and LVO system, with and without air assistance, and one control. LVO applications showed similar control levels as conventional applications. The use of air assistance increased the efficiency of conventional application and reduced the efficiency of LVO applications.
\end{abstract}

Key words: Spraying, weeds, control efficiency

1 Acadêmico de Agronomia da Universidade Estadual do Norte do Paraná, UENP. E-mail: emerson_teixeira20@yahoo.com.br; jethrosipe@hotmail.com; ulisses.gandolfo@hotmail.com

2 Mestrandos em Agronomia pela Universidade Estadual de Londrina, UEL. E-mail: jfoliveira79@yahoo.com.br

3 Prof. Adjunto do Curso de Agronomia da Universidade Estadual do Norte do Paraná, UENP. E-mail: gandolfo@ffalm.br; robosipe@ffalm.br

* Autor para correspondência 


\section{Introdução}

O setor agrícola é responsável por suprir a crescente demanda de alimentos e energia com sua produção, principalmente no fornecimento de matérias primas para indústrias ou de alimentos in-natura. Portanto deve aperfeiçoar seu potencial produtivo para atender esta realidade e reduzir os impactos ambientais prejudiciais.

Fatores como o desenvolvimento econômico mundial ligado ao grande crescimento populacional e os elevados padrões de consumo, estão tornando a água um bem escasso, devido à atuação do homem de maneira despreocupada com o uso de recursos naturais e a poluição ambiental (REIN JUNIOR; FIGUEIREDO; CUNHA JUNIOR, 2008).

Sapadotto (2007) conclui que o grande desafio dos profissionais da agricultura, nos próximos anos, é conciliar a produção agrícola, pecuária, florestal e agroindustrial com os preceitos de responsabilidade social e ambiental.

As aplicações de agroquímicos utilizadas nas principais culturas consomem cerca de 32 milhões de metros cúbicos de água por ano, com projeções de dobrar o consumo no ano de 2017, caso seja mantida a tecnologia de aplicação atual (GANDOLFO et al., 2007). A utilização de micronizadores rotativos possibilitou a utilização de menores volumes de calda no controle $P$. includens na cultura da soja (17 L.ha ${ }^{-1}$ ), produzindo gotas de maior homogeneidade, com menor perda de tempo nos reabastecimento do equipamento, sem prejudicar estatisticamente a porcentagem de cobertura em relação a aplicações hidráulicas de 100 e 50 L.ha-1 $^{-1}$ (OLIVEIRA, 2008).

Camargo (2004) obteve controle eficiente da ferrugem da soja em aplicações aéreas preventivas de fungicidas em BVO (baixo volume oleoso) comparando taxas de aplicação de 8, 12 e 15 L.ha $^{-1}$. Esta mesma tecnologia foi testada em aplicação terrestre utilizando 40 L.ha ${ }^{-1}$ comparada a uma aplicação convencional de 120 L.ha ${ }^{-1}$, com assistência de ar no controle de ferrugem da soja. Os resultados não indicaram diferenças entre os níveis de controle do patógeno e a produção da cultura nos distintos tratamentos (CHRISTOVAM, 2009).

Bracamonte, Loeck e Pinto (1999) afirmam que a redução do volume de calda diminui o custo de operação do tratamento e aumenta a eficiência operacional no campo, possibilitando um melhor gerenciamento do tempo nas operações agrícolas.

Considerando a necessária redução dos elevados volumes de água atualmente utilizados na agricultura e a viabilização econômica das aplicações com a máxima eficiência no controle dos agentes de danos, a tecnologia que permita que as aplicações possam ser realizadas com menores volumes de água, com maior rapidez e menor custo e, ainda, com a garantia de eficiência biológica, poderia contribuir com os avanços do setor agrícola, para uma maior competitividade agrícola e sustentabilidade ambiental.

O objetivo deste trabalho foi avaliar a eficiência do controle de plantas invasoras nas aplicações terrestres com e sem assistência de ar, utilizando volumes convencionais de pulverização comparados com baixo volume oleoso (BVO).

\section{Material e Métodos}

O trabalho foi conduzido no campus experimental da Universidade Estadual do Norte do Paraná - Campus Luiz Meneghel, Bandeirantes PR, com apoio do NITEC - Núcleo de Investigação em Tecnologia de Aplicação de Agrotóxicos e Máquinas Agrícolas.

$\mathrm{Na}$ aplicação foi utilizado um pulverizador Jacto, modelo Falcon Vórtex, com capacidade nominal de $600 \mathrm{~L}$ de calda e barra de $14 \mathrm{~m}$ de comprimento, acoplado a um trator operando a velocidade de 1,5 $\mathrm{m} \cdot \mathrm{s}^{-1}$.

Os tratamentos comparados foram aplicações convencionais e em BVO, ambas com e sem assistência de ar, além de uma testemunha que não recebeu aplicação. 
Para as aplicações convencionais, as pontas de pulverização utilizadas foram de jato plano comum modelo AXI 11002, espaçadas em 0,5 m, operando com pressão de $414 \mathrm{kPa}$ e vazão correspondente de 0,91 L. min $^{-1}$. Estas pontas foram montadas na barra do lado direito do pulverizador e durante a operação o registro apenas do mesmo lado da barra foi aberto. Os herbicidas utilizados foram glyphosate na dose de 1080 g.ha-1 de equivalente ácido e 2,4 - D amina na dose de $860{\mathrm{~g} . h a^{-1}}^{-1 e}$ ingrediente ativo.

Do lado esquerdo da barra do pulverizador foi montado o sistema para aplicação em BVO. Este sistema constou da instalação de 4 micronizadores elétricos modelo Turbotrator, espaçados em 1,4 m, acionados por um controlador elétricos modelo Turbo Controlador II, ajustado para a rotação dos micronizadores na posição "Grossa". A pressão de trabalho do circuito hidráulico neste sistema foi de $138 \mathrm{kPa}$, utilizando como limitador de vazão a ponta D-2/25, o que determinou uma vazão de cada ponta de $0,38 \mathrm{~L} \cdot \mathrm{min}^{-1}$ e taxa de aplicação correspondente a 20 L.ha $^{-1}$. Os herbicidas utilizados foram glyphosate na dose de 1080 g.ha-1 de equivalente ácido e 2,4 $\mathrm{D}$ amina na dose de $860 \mathrm{~g}$.ha ${ }^{-1}$ de ingrediente ativo. A calda foi preparada com óleo vegetal de soja, com dose proporcional de 1,0 L.ha-1 somado a 0,2 L.ha-1 do emulsificante Agral, conforme recomendação de Vilela (2007).

A regulagem do sistema de assistência de ar, para as duas formas de aplicação descritas (convencional e BVO) foi estabelecida para o nível de ventilação número 8 , correspondente a $80 \%$ da capacidade máxima de ventilação do sistema. Para os tratamentos sem assistência de ar o mesmo foi apenas desligado.

$\mathrm{O}$ delineamento experimental empregado foi inteiramente ao acaso com parcelas de $48 \mathrm{~m}^{2}$, sendo $6 \mathrm{~m}$ de largura por $8 \mathrm{~m}$ de comprimento. A aplicação ocorreu em pós-emergência, sendo que no momento da aplicação havia a predominância de Digitaria insularis (L.) Fedde. e Commelina benghalensis L., que se encontravam em inicio de desenvolvimento vegetativo, com 15 e $20 \mathrm{~cm}$ de altura respectivamente.

As avaliações de eficiência foram realizadas visualmente aos 07, 14, 21 e 28 dias após aplicação (d.a.a), mediante a comparação com a testemunha, estabelecendo-se porcentagens de controle. Os dados obtidos foram submetidos à análise de variância e suas médias ao teste Tukey em nível de $5 \%$.

\section{Resultados e Discussão}

\section{Controle de Commelina benghalensis L.}

Aos 7 d.a.a. os resultados de aplicação com sistema convencional com assistência de ar apresentou maior percentual de controle (Tabela 1), seguido pelas aplicações com sistema BVO e convencional, ambos sem assistência de ar que não diferenciaram entre si. O sistema BVO com assistência de ar que apresentou percentual de controle inferior (56,25\%).

Aos 14 d.a.a. houve melhora no controle em todos os tratamentos com os diferentes equipamentos testados. Novamente a aplicação com sistema BVO com assistência de ar obteve nível de controle estatisticamente inferior, com percentual de $63,75 \%$. Os demais tratamentos apresentaram níveis de controle acima dos $77 \%$.

A mesma análise, aos 21 d.a.a, revelou um comportamento semelhante às avaliações anteriores, ou seja, um nível de controle crescente com o tempo, superando os $83 \%$ de controle, exceto a aplicação com o sistema BVO com assistência de ar, que além de apresentar nível de controle significativamente inferior aos demais, também mostrou redução da eficiência no controle das plantas daninhas em relação à avaliação anterior $(61,25 \%)$.

A avaliação aos 28 d.a.a permitiu observar que as aplicações com sistema convencional com assistência de ar e BVO sem assistência de ar não diferiram estatisticamente entre si e mostraram 
níveis de controle acima de 96\%. A aplicação com sistema convencional sem assistência de ar foi significativamente inferior às anteriores, porém com um nível de controle ainda elevado (87,5\%). Já a aplicação com sistema BVO com assistência de ar foi significativamente inferior a todos os outros, não superando $55 \%$ de controle.

Considerando os valores apresentados, podese observar um efeito crescente no controle para todos os tratamentos, exceto na área que recebeu aplicação em BVO com assistência de ar, o que permitiu uma recuperação da planta tratada e emissão de novas brotações. A menor eficiência de controle nas aplicações com sistema BVO associada à assistência de ar pode ser atribuída ao fato de que as gotas menores produzidas no sistema BVO foram movidas para outro local que não a área em tratamento, por ação física devido à deflexão do ar no solo produzida pelo equipamento em uso, um efeito característico das aplicações realizadas com assistência de ar em áreas de baixa densidade foliar.

Desta forma, as aplicações convencionais dos produtos testados podem ser substituídas pelas aplicações em BVO sem prejuízo na qualidade de controle desta planta daninha, desde que, se aplicada em áreas com baixa densidade foliar, não seja associada ao uso da assistência de ar.

Tabela 1. Médias das porcentagens de eficiência do Controle de Commelina benghalensis L. aos 7, 14,21 e 28 dias após a aplicação.

\begin{tabular}{lrrrlrlrl}
\hline \multicolumn{1}{c}{ Tratamento } & \multicolumn{8}{c}{ Commelina benghalensis L. } \\
\cline { 2 - 8 } & \multicolumn{1}{c}{ 7 DAA } & 14 DAA & 21 DAA & 28 DAA & \\
\hline Convencional com ar & 78,75 & $\mathrm{~A}$ & 87,5 & $\mathrm{~A}$ & 93,75 & $\mathrm{~A}$ & 97,25 & $\mathrm{~A}$ \\
BVO sem ar & 72,5 & $\mathrm{~B}$ & 82,5 & $\mathrm{AB}$ & 87,5 & $\mathrm{AB}$ & 96,5 & $\mathrm{~A}$ \\
Convencional sem ar & 72,5 & $\mathrm{~B}$ & 77,5 & $\mathrm{~B}$ & 83,75 & $\mathrm{~B}$ & 87,5 & $\mathrm{~B}$ \\
BVO com ar & 56,25 & $\mathrm{C}$ & 63,75 & $\mathrm{C}$ & 61,25 & $\mathrm{C}$ & 55 & $\mathrm{C}$ \\
Testemunha & 0 & $\mathrm{D}$ & 0 & $\mathrm{D}$ & 0 & $\mathrm{D}$ & 0 & $\mathrm{D}$ \\
\hline C.V. & 5,47 & $\%$ & 4,31 & $\%$ & 5,75 & $\%$ & 3,17 & $\%$ \\
\hline
\end{tabular}

As médias seguidas da mesma letra nas colunas não diferem entre si (Tukey $-5 \%$ de probabilidade).

\section{Controle de Digitaria insularis (L) Fedde}

Aos 7 d.a.a todos os tratamentos diferenciaramse estatisticamente entre si (Tabela 2), com o melhor resultado atribuído aos sistema convencional com assistência de ar, que obteve controle superior a $82 \%$, seguido da aplicação convencional sem assistência de ar com 70\%. As demais mostraram níveis de controle inferiores a $50 \%$, sendo que o BVO com assistência de ar não chegou a 30\%.

Avaliações aos 14 d.a.a. mostraram acréscimo no percentual de controle para todos os tratamentos. $\mathrm{O}$ de maior eficiência foi a aplicação convencional com assistência de ar (88,75\%), seguido pela aplicação convencional sem assistência de ar (82,5\%). Houve também melhoria do nível de controle da aplicação com sistema BVO sem assistência de ar, superando $67 \%$, e novamente a aplicação com o sistema BVO com assistência não superou $30 \%$.

Aos 21 d.a.a. o tratamento com aplicação convencional com assistência de ar manteve o maior nível de eficiência no controle $(93,75 \%)$. As aplicações em BVO e convencional sem assistência de ar, não se diferenciaram entre si, com eficiência no controle superior a $81,25 \%$. A aplicação em BVO com assistência de ar manteve o mesmo nível de controle da avaliação anterior (30\%).

Nas avaliações aos 28 dias foi observada uma igualdade estatística entre os tratamentos, com eficiência superior a $96 \%$, exceto para o BVO com assistência de ar que mostrou redução na 
eficiência de controle não superando $20 \%$, tal como ocorreu para Commelina benghalensis L., analisada anteriormente. Para este tratamento foi observada uma recuperação foliar da planta com o passar do tempo, caracterizando eficiência insuficiente no controle para esta técnica de aplicação.

Como já citado anteriormente, neste caso foi observada também uma baixa densidade foliar das plantas tratadas, o que poderia causar uma redução do nível de controle quando associadas às gotas menores do sistema BVO com o uso de assistência de ar no equipamento de aplicação, reduzindo os depósitos de herbicida nas plantas alvo devido à deflexão do ar no solo e arrastamento das gotas para outro local.
Apesar dos herbicidas usados apresentarem reconhecida e diferenciada eficiência para cada uma das espécies de plantas daninhas analisadas, foi observado um comportamento semelhante para cada técnica de aplicação independente da planta daninha considerada, evidenciando uma diferença no nível de eficiência em função da técnica de aplicação utilizada.

Isto pode indicar uma necessidade de melhores estudos, caracterização e descrição das técnicas que podem ser empregadas na aplicação destes e de outros agroquímicos para fins de controle de plantas invasoras, com tanta importância quanto à ação específica do produto na eficiência de controle destas plantas.

Tabela 2. Médias das porcentagens da eficiência do controle de Digitaria insularis (L) Fedde, aos 7, 14, 21 e 28 dias após a aplicação.

\begin{tabular}{|c|c|c|c|c|c|c|}
\hline \multirow{2}{*}{ Tratamento } & \multicolumn{6}{|c|}{ Digitaria insularis (L.) Fedde } \\
\hline & 7 DAA & 14 DAA & 21 DAA & & 28 DAA & \\
\hline Convencional com ar & 82,5 a & $88,75 \mathrm{~A}$ & 93,75 & $\mathrm{~A}$ & 99 & A \\
\hline Convencional sem ar & $70 \mathrm{~b}$ & 82,5 B & 85 & $\mathrm{~B}$ & 96,5 & A \\
\hline BVO sem ar & $42,5 \mathrm{c}$ & $67,5 \mathrm{C}$ & 81,25 & B & 96,5 & A \\
\hline BVO com ar & $25 \mathrm{~d}$ & $30 \mathrm{D}$ & 30 & $\mathrm{C}$ & 20 & B \\
\hline Testemunha & $0 \quad \mathrm{e}$ & $0 \mathrm{E}$ & 0 & $\mathrm{D}$ & 0 & $\mathrm{C}$ \\
\hline C.V. & $7,19 \%$ & $5,4 \%$ & 5,94 & $\%$ & 3,96 & $\%$ \\
\hline
\end{tabular}

As médias seguidas da mesma letra nas colunas não diferem entre si (Tukey - 5\% de probabilidade).

Uma vez que Camargo (2004) e Christovam (2009) já haviam utilizado a tecnologia BVO, respectivamente, em aplicações aéreas e terrestres para controle de doenças na soja com sucesso, o uso desta técnica com objetivo de otimizar recursos ambientais e econômicos, tais como a água e o rendimento da máquina, inclusive na aplicação de herbicidas, também é possível de ser executada com níveis de eficiência semelhantes ao sistema convencional.

\section{Conclusões}

As aplicações com sistema BVO sem assistência de ar mostraram níveis de controle semelhantes às aplicações convencionais.

O uso da assistência de ar elevou a eficiência das aplicações convencionais e reduziu a eficiência das aplicações em BVO. 


\section{Referências}

BRACAMONTE, E. R.; LOECK, A. E.; PINTO, J. O. Eficiência do herbicida sethoxydim em função do volume de calda no controle de papua (Brachiaria plantaginea (Link.) Hitch.) na cultura da soja. Revista Brasileira de Agrociência, Pelotas, v. 5, n. 1, p. 60-63, jan./abr. 1999.

CAMARGO, T. V. Aplicação aérea e terrestre de fungicidas no controle de ferrugem da soja. In: WORKSHOP BRASILEIRO SOBRE A FERRUGEM ASIÁTICA, 1., 2005, Uberlândia. Anais... Uberlândia: EDUFV, 2005. p. 181-191.

CHRISTOVAM, R. D. S. Assistência de ar e aplicação em baixo volume no controle da ferrugem asiática da soja (Phakopsora pachyrhizi Sydon \& Sydon). 2008. Tese (Mestrado em Agronomia) - Faculdade de Ciências Agronômicas. Universidade Estadual Paulista Júlio de Mesquita Filho, Botucatu.

GANDOLFO, M. A.; SAUER, A. V.; JESUS, J. T.; AFONSO, M. Demanda de água atual e futura nas aplicações de agroquímicos. Cuiabá: Associação Brasileira de Recursos Hídricos, 2007. CD-ROM.
OLIVEIRA, J. R. G. D. Cobertura da cultura da soja e deposição de inseticida aplicado com e sem adjuvante e diferentes equipamentos e volumes de calda. 2008. Tese (Mestrado em Agronomia) - Faculdade de Ciências Agrárias e Veterinárias. Universidade Estadual Paulista Júlio de Mesquita Filho, Jaboticabal.

REIN JUNIOR, O.; FIGUEIREDO, H. L.; CUNHA JUNIOR, C. A. S. C. Recursos hídricos e hidrogeração. In: SIMPÓSIO INTERNACIONAL DE CIÊNCIAS INTEGRADAS DA UNAERP, 1., 2008, Guarujá. Anais... Guarujá: UNAERP, 2008. p. 10.

SAPADOTTO, C. A. Agricultura Brasileira: importância, perspectivas e desafios para os profissionais dos setores agrícolas e florestais. CNPMA/EMBRAPA. 2007. Disponível em: <http://www.cnpma.embrapa.br/ down_hp/354.pdf>. Acesso em: 27 mar. 2009.

VILELA, M. Fatores de sucesso no sistema BVO. In: BORGES, D. L. (Org.). Tecnologia de aplicação de defensivos agrícolas II. Passo Fundo: Plantio Direto Eventos, 2007. Cap. 10, p. 129-138. 